\title{
SMOOTH-ROCKING OSCILLATOR UNDER NATURAL ACCELEROGRAMS
}

\author{
Blerta Lipo ${ }^{1}$, Gianmarco de Felice ${ }^{2}$ \\ ${ }^{1}$ University Roma Tre \\ e-mail: blerta.lipo@uniroma3.it \\ ${ }^{2}$ University Roma Tre \\ e-mail: gianmarco.defelice@uniroma3.it
}

Keywords: Rocking, masonry, seismic analyses.

\begin{abstract}
Following the work by Psycharis [1], in this paper we study the dynamic behavior of a rocking block resting on an elastic Winkler-type foundation for the out-of-plane seismic assessment of masonry walls. The equations of motion are derived and implemented for the simple smooth-rocking block and the calibration of the Winkler-type foundation stiffness is carried out according to the experimental results available in the literature. The dynamic response under natural seismic records is evaluated and the effect of the stiffness on the overall response is analyzed for different natural accelerograms. Both demand and capacity are calculated with reference to the kinetic and potential energies of the system and different intensity measures are considered with the aim of identifying the most representative one for the rocking motion. Finally, a comparison between non-linear time history analyses and a possible energy-based approach is discussed.
\end{abstract}

\section{INTRODUCTION}

The out-of-plane rocking of masonry walls is the most recurrent mechanism triggered by earthquake shocks in historical constructions. This is why, increasing interest is shown in the study of this type of mechanism for the purpose of predicting existing buildings responses during a seismic event. Even though this behavior is complicated to capture, it is seen that could be well represented by means of a single degree of freedom oscillator. This simple approach has been often preferred to more complex models, which, while having greater chance to better grasp the response, may present inherent difficulty in the choice of inputs parameters.

The first major study on the behavior of slender structures subjected to rocking motion was carried out by Housner [2] in the well known "The behavior of inverted pendulum structures 
during earthquakes". He described the rocking motion with a model that included a linear force-displacement relation for the block and damping concentrated at impacts. Thanks to this simple but powerful approach, which proved able to reproduce the main feature of rocking behavior, Housner was the first to point up that a gravity structure can exhibit significant capacity towards overturning by tilting/rocking. Recently it has been demonstrated that the same model may be extended also to study the response of a wider set of mechanisms developed by masonry constructions (Mauro et al. [3]). After Housner fundamental study, many other authors have defined different force-displacement relationships for describing the motion of the block from the triggering of the mechanism until the out-of-plane collapse. In 2002 Doherty et al. [4] presented a trilinear curve used to reproduce the nonlinear forcedisplacement relationship obtained experimentally for unreinforced brick masonry walls. In Shawa et al. [5], starting from existing rocking models, a new three-branch model is formulated which takes into account the asymmetry resulting from the restraint provided by transverse walls and the out-of-plumb of the block. Ferreira et al. [6] proposed a simplified analytical approach based on a linearized four-branch model used to characterize the response of stone masonry sacco walls in terms of overturning moment-rotation relationship. The models adopted so far in literature proved able to reproduce the rocking of masonry blocks tested in laboratory. Nevertheless, their main drawback depends on the fact that, in order to increase the accuracy, the number of parameters is increased and that these parameters may lack a physical meaning.

In this paper, a smooth single degree of freedom model is presented for reproducing the rocking behavior of masonry walls. With respect to the Housner model, only one parameter is added which consists in the stiffness of the foundation represented with a set of unilateral springs.

\section{GENERAL FORMULATION OF THE PROBLEM}

As mentioned before, the most known rocking block in literature is the one developed by Housner [2] that can only oscillate alternatively around the two pivot points $\mathrm{O}$ and $\mathrm{O}$ ' (Figure 1, a). Therefore it is assumed that the static friction coefficient between the block and its base is high enough for sliding to be negligible, verified hypothesis for adequately slender objects.

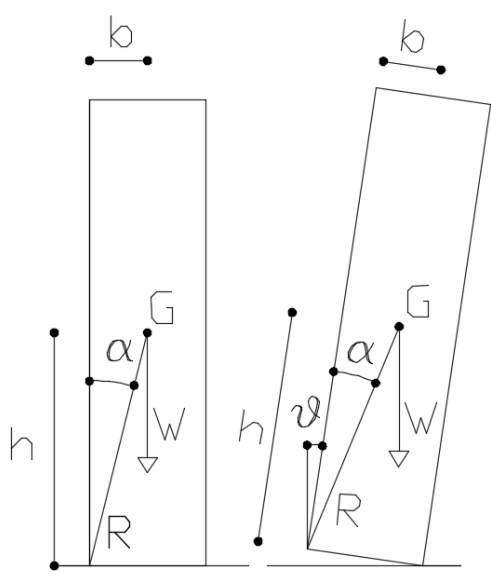

a)

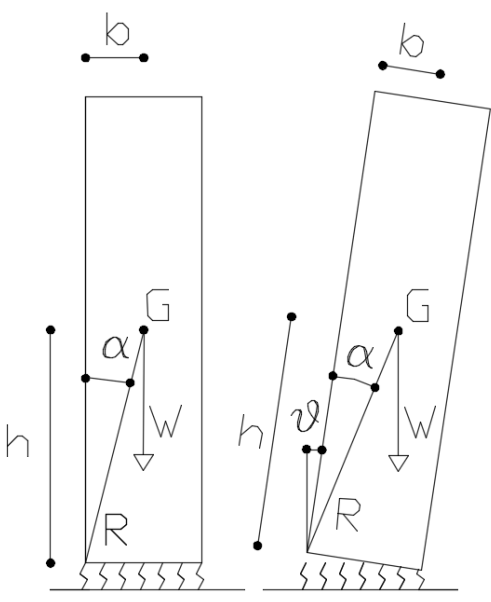

b)

Figure 1. Comparison between the rigid model (a) and the smooth-rocking model (b). 
This inverted pendulum has only one degree of freedom, the tilting angle $\theta$ from the vertical position and its motion under a horizontal ground acceleration $\ddot{u}_{\mathrm{g}}$ can be described by equation 1. The significant parameters are the moment of inertia of the block $\mathrm{I}_{0}$ about the point 0 , the restoring moment $M_{R}$, that depends on wall geometry and self-weight $W=g m$, and the ground acceleration $\ddot{u}_{\mathrm{g}}$. Symbols are depicted in Figure 1.

$$
I_{0} \ddot{\theta}+M_{R}(\theta, \operatorname{sign}(\theta))=-m \ddot{u}_{g} R \cos (\alpha \operatorname{sign}(\theta)-\theta)
$$

The block will initiate rocking only if the ground acceleration is greater than a threshold value depending on its slenderness $\alpha$. During rocking, motion is interrupted by subsequent impacts at the pivot points with consequently dissipation of energy [7]. In this paper, such phenomenon is treated as in Housner [2], by reducing the angular velocity when the block passes the resting position, as shown in eq. (2).

$\dot{\theta}^{+}=\eta \dot{\theta}^{-}$

where $\dot{\theta}^{+}$and $\dot{\theta}^{-}$are the angular velocities before and after impact, respectively. The equation of motion (1), together with the impact treatment, equation (2), have been implemented in Matlab R2015b and integrated numerically using a Runge-Kutta algorithm with integration step and tolerances sufficiently appropriate to guarantee an adequate accuracy of the solution.

\subsection{The smooth-rocking moment-rotation relationship}

Various experimental evidences ([4]) have shown that the actual response of masonry walls loaded out-of-plane may be far different from the rigid (bilinear) moment-rotation relationship assumed by Housner [2]. More precisely, when dealing with brick or stone masonries, a smoother response has been observed ([6]). In this paper, in order to better simulate the actual behavior of masonry walls, a smooth restoring moment-rotation is obtained by considering a rigid block resting on a Winkler-type foundation (Figure 1b). This approach makes it possible to introduce only one additional parameter with respect to those defined by Housner, precisely the stiffness $\mathrm{k}$ of the springs on which the wall is resting.

The equations relative to the Winkler-type foundation, derived in Shawa et al [6] for the case of one-sided rocking, are here reported for the two-sided case (eq. 3) where $M_{R}$ is the restoring moment, $\theta_{\mathrm{cr}}$ is the relative rotation corresponding to lift-off of the block, the other symbols have the same meaning as in Housner [2] (Figure 1b).

$$
\begin{array}{ll}
M_{R}=W R \sin (\alpha \operatorname{sign}(\theta)-\theta)-W b \operatorname{sign}(\theta)+\frac{2}{3} k b^{3} \theta & \text { for } \theta \leq \theta_{c r} \\
M_{R}=W R \sin (\alpha \operatorname{sign}(\theta)-\theta)-\frac{1}{6} k \theta \sqrt{\left[\frac{2 W}{k \theta \operatorname{sign}(\theta)}\right]^{3}} \quad \text { for } \theta>\theta_{c r} \\
\theta_{c r}=\frac{W}{2 b^{2} k} &
\end{array}
$$


The vertical component of the displacement is neglected during the motion of the block and the rotations instead are considered sufficiently small. In order to model the inability of the ancient mortar to sustain tensile stresses, it is assumed that the block is just resting on the springs, without any bond between them. In particular, the moment-rotation curve presents two fictitious branches corresponding to the case where the coupling of the springs is perfectly reagent $\left(\theta \leq \theta_{\mathrm{cr}}\right)$, and the case where contact is lost in one of the base edges and a limited portion only of the base section is reacting $\left(\theta>\theta_{\text {cr }}\right)$.

In Figure 2 the moment-rotation relationship for a block having $2 \mathrm{~b}=0.25 \mathrm{~m}$ and $2 \mathrm{~h}=3 \mathrm{~m}$ and a specific weight of $12.06 \mathrm{kN} / \mathrm{m}^{3}$ is presented, comparing the results for different values of the stiffness $\mathrm{k}$. For finite values of $\mathrm{k}$, a linear behavior is exhibited until $\theta \leq \theta_{\mathrm{cr}}$, then the response suddenly curves to the maximum allowable restoring moments and then goes to an almost linear branch with negative stiffness until the ultimate rotation is reached. Noteworthy, the maximum rotation the block can bear without overturning is not reached for $\theta=\alpha$ as in the Housner [2] oscillator but it also depends on the stiffness of the springs and is derived here numerically starting from equation $3 \mathrm{~b}$ ). When the foundation springs are very stiff, the block is expected to behave as if it was rocking on a rigid foundation, therefore, for $\mathrm{k} \rightarrow \infty$, the second branch of the restoring moment-rotation curve $(\theta>\theta \mathrm{cr})$ tends to WR $\sin (\alpha-\theta)$ and the first branch tends to infinity in the vertical direction.

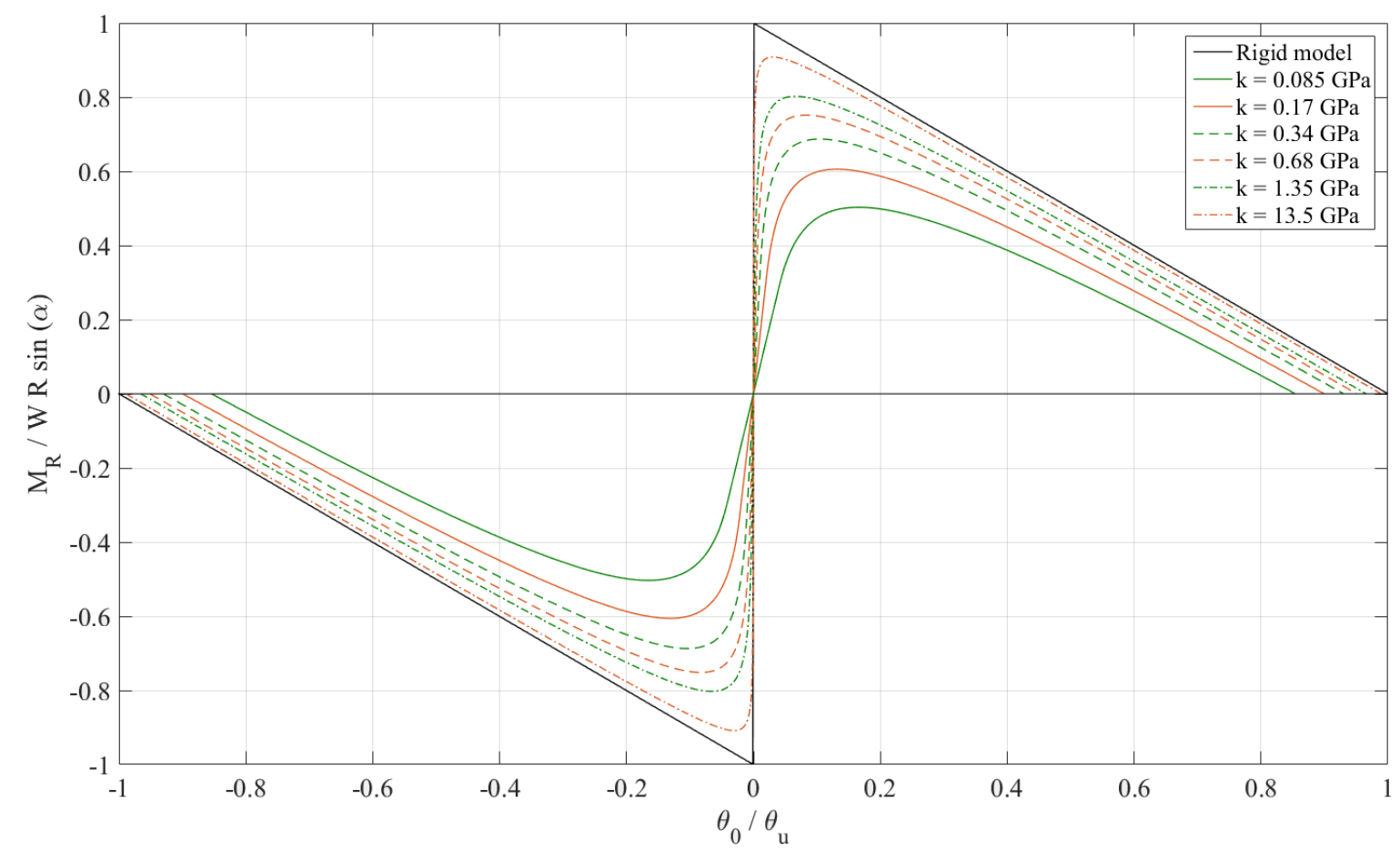

Figure 2. Moment rotation variation for different stiffness values.

It is worth underlining that the curves represented in Figure 2 by varying the values of $\mathrm{k}$ resemble those obtained by de Felice [8] for multi-leaf walls exhibiting failure for disaggregation because of the absence of connection throughout the thickness of the wall. Accordingly, the introduction of the parameter $\mathrm{k}$ would make it possible to fictitiously account for any losses of monoliticity of the wall by reducing its restoring moment and ultimate displacement capacity. 
The free oscillatory motion of a block occurs with a period which, as known, is a function of the maximum angle of rotation that is reached during the motion. In Figure 3, there are shown the values of the period of a block, obtained numerically according to the maximum rotation $\theta_{\mathrm{u}}$ referring to different value of stiffness $\mathrm{k}$. For initial rotation $\theta_{0}<\theta_{\mathrm{cr}}$ the period $\mathrm{T}$ is constant and corresponds to oscillations without partialization of the base joints. When approaching the overturning rotation periods tends to infinity.

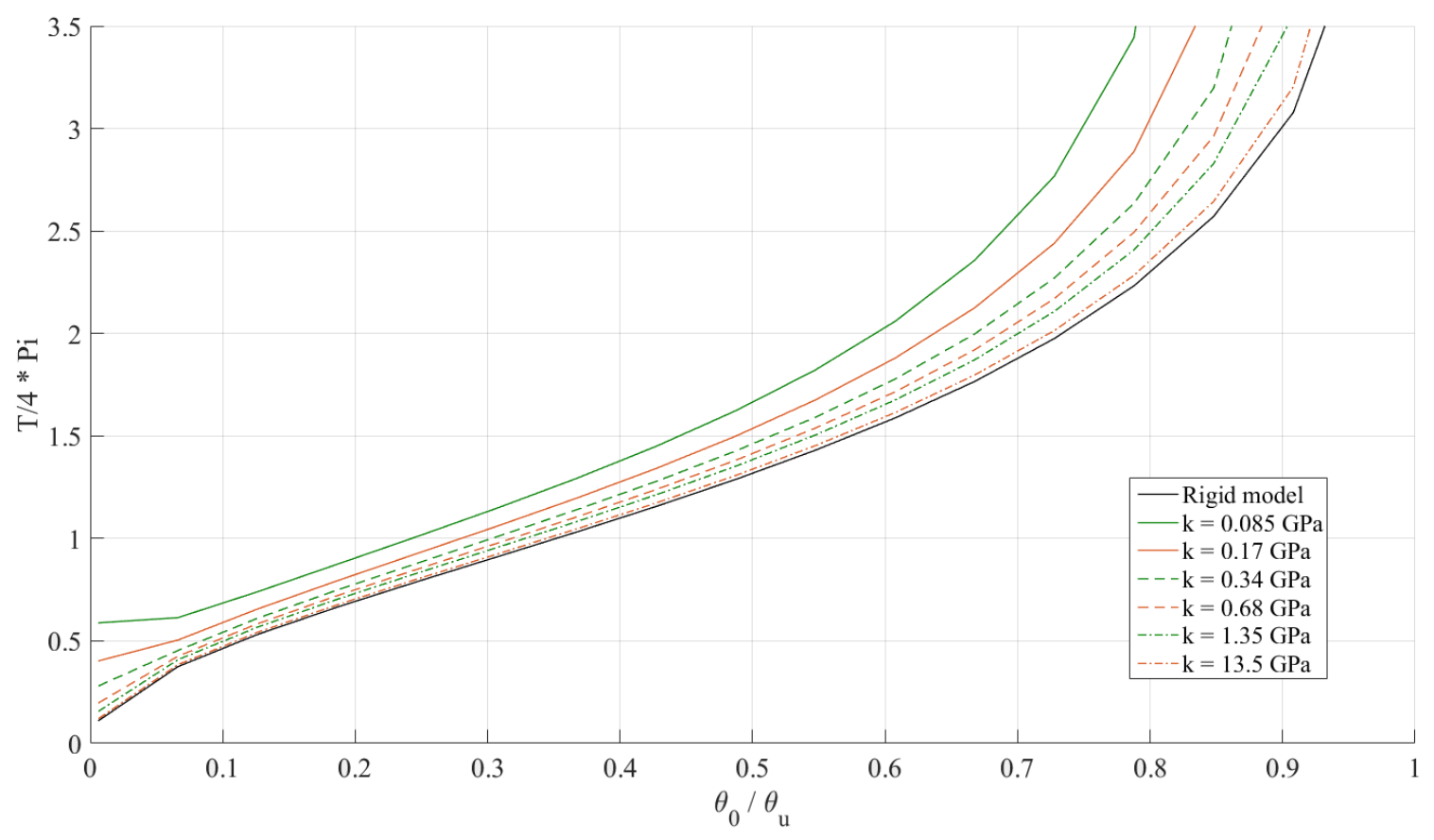

Figure 3. Period T of block laying in different stiffness layer rocking with amplitude $\theta_{0}$.

\section{CALIBRATION OF THE STIFFNESS K BASED ON EXPERIMENTAL DATA}

The equations of motions (eq. 3 and eq. 2) have been implemented in Matlab R2015b and integrated using the ode solver. Different integration options have been considered in order to better evaluate the response of the block. Aiming at investigating the feasibility of the proposed approach some comparisons with experimental data are briefly reported.

\subsection{Static tests}

The first comparison is made with respect to the experimental results presented by Ferreira et al. [6] which have performed static tests on six full-scale $(1.3 \mathrm{~m} \times 0.65 \mathrm{~m} \times 2.50 \mathrm{~m})$ unreinforced regular sacco stone masonry specimens subjected to horizontal out-of-plane loading and three different vertical loadings $\mathrm{N}$. Here are compared the results of the wall without force on the top $(\mathrm{N}=0 \mathrm{kN})$ undergoing a uniform pressure applied by means of an airbag. Among the others setups tested, the wall selected exhibited a cracking pattern which is more in line with the assumption made in the present work, i.e characterized by a single crack at the base, while the other tests yielded to a bending-rocking pattern. The results reported in Figure 4, confirms that the proposed approach is able to fit reasonably well the response of a masonry wall undergoing static overturning forces. The image representing the cracking pattern is taken from [12]. 


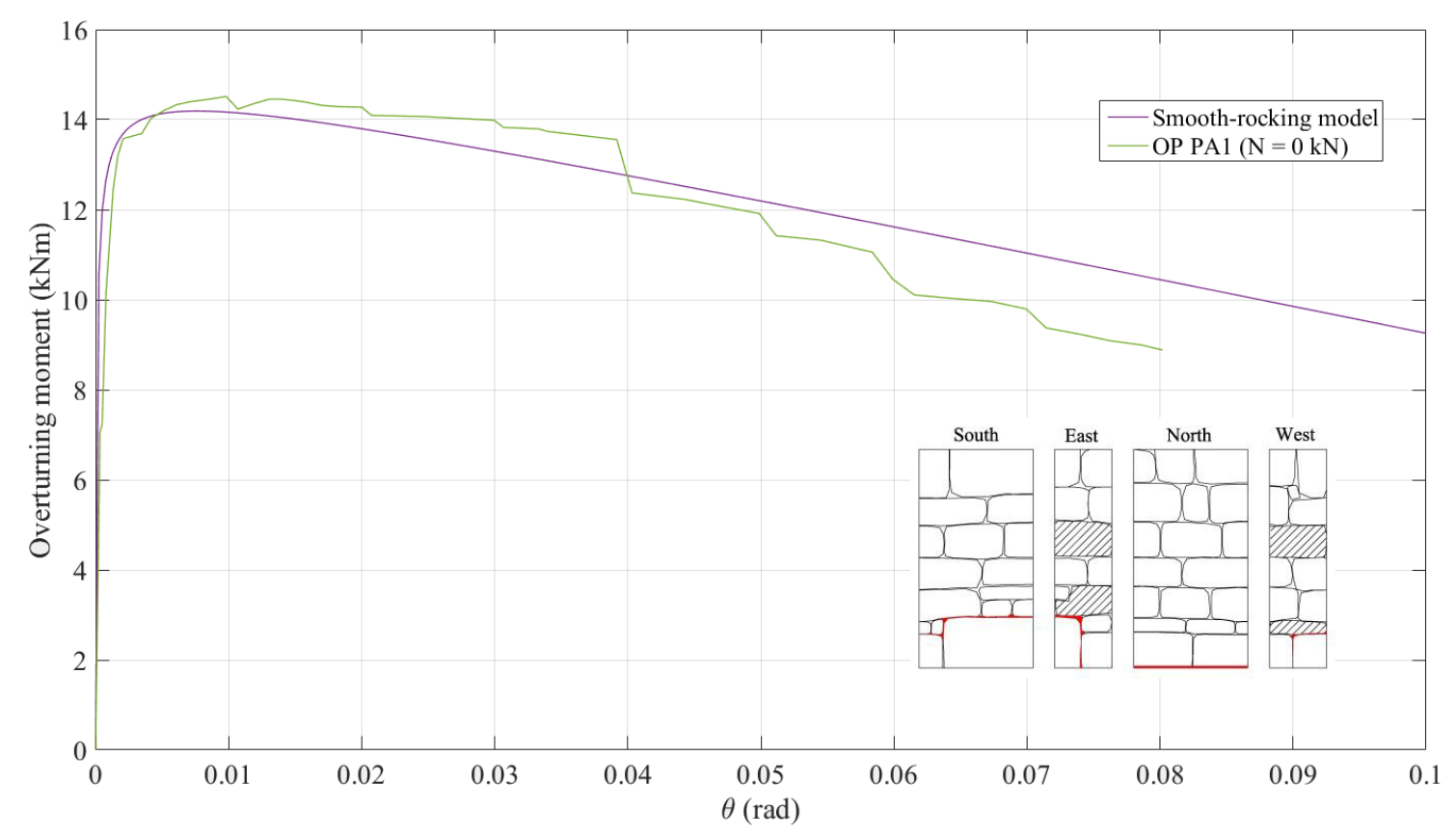

Figure 4. Overturning moment-rotation curves for airbag tests compared to the smooth model.

\subsection{Dynamic tests}

El Gawady et al. [9] performed some free rocking experimental tests of walls rocking on foundations made of different materials. Each block was initially tilted to an angle $\theta_{0}$, held still, and then released with zero angular velocity at time $t=0$, thereafter rocking back and forth freely. In order to validate the equations of motion derived for free vibrations, we are comparing here the results for three different interface material: concrete, 4-layer rubber and timber for a block with aspect ratio 5. The parameters to calibrate for fitting the curves are two: the stiffness $\mathrm{k}$ and the coefficient of restitution $\eta$ because they both depend on the foundation material. The parameter $\mathrm{k}$ has been calibrated referring to the half-period rotation graphics starting from typical stiffness values for each interface material. Then the coefficient of restitution $\eta$ is calibrated in order to better fit the rest of the experimental data. The results in figure 5 demonstrate that the proposed model can fit rationally well the free vibration of blocks resting on foundation made by different materials. 


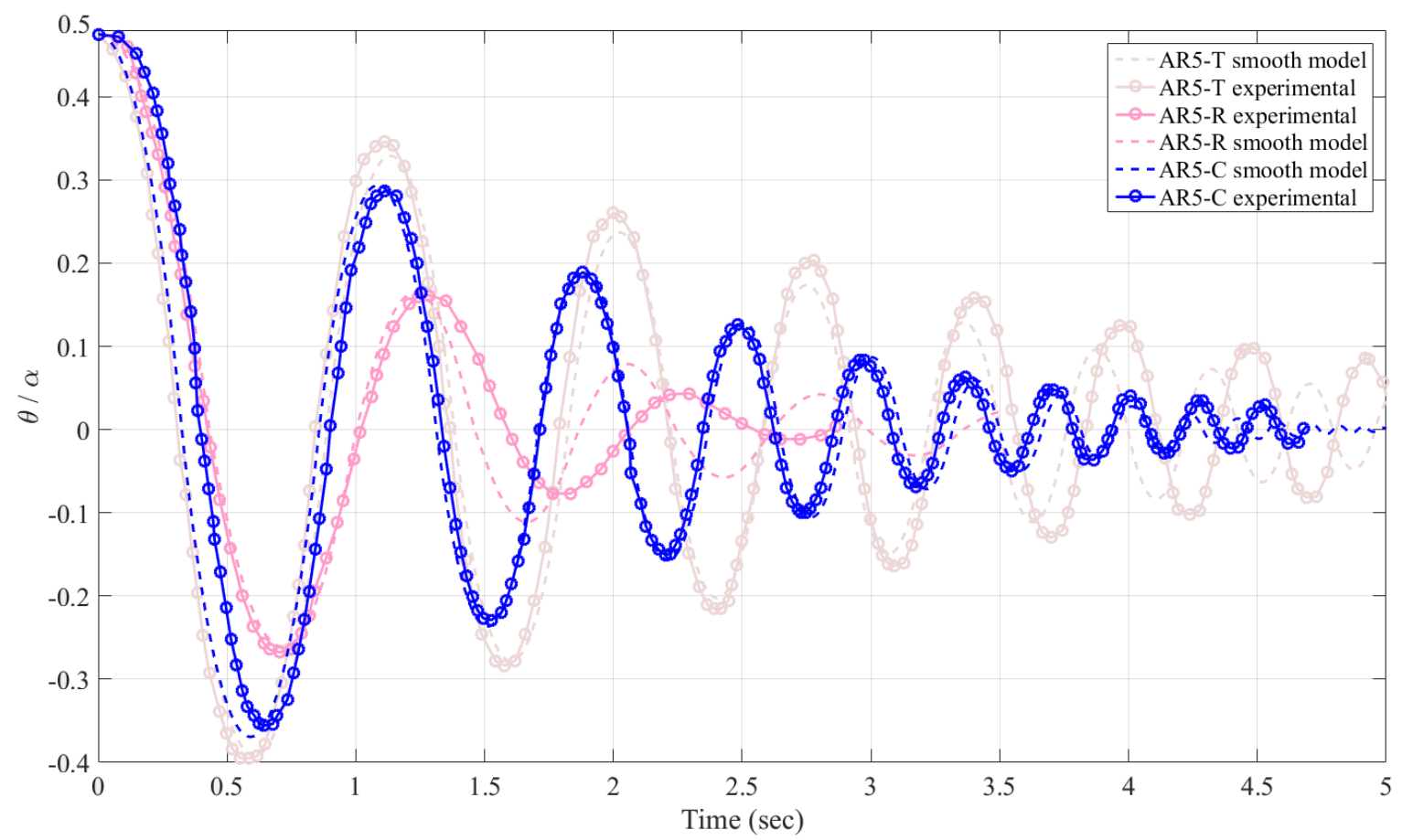

Figure 5. Comparison between experimental results and smooth model free oscillations on different interface material.

\section{ASSESSMENT STRATEGIES FOR THE SMOOTH-ROCKING BLOCK}

For the purpose of understanding how actual walls behave during the rocking motion, in this section we compare the non-linear time-history analyses of the smooth-rocking block with Italian code-procedures [10] and a possible energy-based approach [11]. Given the strong numerical integration sensitivity, a parametric analysis varying from time to time the accelerograms is performed. The latter varies in a purely random way by multiplying both the time-axis and the acceleration-axis with random coefficients belonging to a normal distribution having $3 \%$ coefficient of variation and unity mean [5]. Four walls, having (2b, $2 \mathrm{~h})=(0.125,1.5),(0.20,3.0),(0.25,3.0),(0.375,3.0) \mathrm{m}$, are considered. The four accelerograms of table 1 are used, whose amplitudes are scaled with ten coefficients varying from 0.2 to 2.0 .

\begin{tabular}{llllllll}
\hline Earthquake & Year & Mw & Station & Soil type & Record & PGA $(\mathrm{g})$ & PGV (m/s) \\
\hline Irpinia & 1980 & 6.9 & Bagnoli Irpino & A & BagnirWE & 0.167 & 0.377 \\
Umbria-Marche & 1997 & 6.0 & Nocera Umbra & B & R1168EW & 0.438 & 0.280 \\
Irpinia & 1980 & 6.9 & Calitri & B & CalitWe & 0.181 & 0.317 \\
Irpinia & 1980 & 6.9 & Sturno & B & SturWE & 0.313 & 0.700 \\
\hline
\end{tabular}

Table 1: Accelerograms selected to perform numerical analyses.

From each dynamic analysis an energy demand/capacity ratio, ED/EC, is obtained as originally proposed by Shawa et al. [5] for the rigid model while closed form solution equations are derived for the smooth model. In particular, the capacity EC is evaluated as the area underneath the restoring-moment - rotation curve and differs from block to block (Fig. 
$6 \mathrm{~b})$. The demand ED is evaluated as the maximum potential energy recorded during the motion if $\theta<\theta_{\mathrm{u}}$, or otherwise, as the sum of potential and kinetic energies evaluated at $\theta=\theta_{\mathrm{u}}$. Moreover, a reduced coefficient of restitution, $\eta=0.85$, which multiplies angular velocity before impact to give angular velocity after impact, is used, based on experimental results. Each dot in the following figures represents the median demand/capacity ratio out of one hundred time histories, in order to account for the scatter of the response. A total of 16000 time history analyses have been performed.

The demand/capacity ratio of the smooth-rocking block is then compared with force-based and displacement based procedures. In particular, for the force-based assessment procedure it is checked ED/EC in comparison to PGA/ $\left(2 \mathrm{a}_{0}{ }^{*}\right)$ where PGA is the Peak Ground Acceleration, 2 is a behavior factor and $\mathrm{a}_{0}{ }^{*}$ is defined in equation 4 , where $\lambda_{0}$ is the static collapse load multiplier of the mechanism at rest and its value is calculated as $\tan (\alpha)$, while $\mathrm{e}^{*}=1$ defines the participating mass and $\mathrm{CF}=1$ is a confidence factor.

$a_{0}^{*}=\frac{\lambda_{0} g}{e^{*} C F}$

As far as regards the displacement-based procedure, the comparison is made between the demand/capacity ratio and the fraction between the spectral demand evaluated for the secant period $\mathrm{S}_{\mathrm{De}}\left(\mathrm{T}_{\mathrm{s}}\right)$ and the displacement capacity $\mathrm{d}_{\mathrm{u}}{ }^{*}$.

Then, a possible energy based verification is considered as initially introduced by Sorrentino et al. in [11]. In this case, the verification is satisfied if the kinetic energy KE, multiplied by 2, is not larger that the area below the skeleton moment-rotation curve (E0) of the rigid block. The kinetic energy of the system is defined considering instead of the translational velocity $\mathrm{v}$, the Peak Ground Velocity (PGV) of each accelerogram reported in table 1. Bearing in mind that the block motion is rotational, its kinetic energy depends on the angular velocity $\dot{\theta}$ instead of the translational one.

The first comparison is made between the smooth block and the rigid one in terms of demand/capacity ratios (Figure 6). As it can be seen from the graph, the smooth model is probably more realistic since it reacts even in the field of small oscillations differently from the rigid block that starts rocking only if it is exceeded a certain threshold. Moreover the smooth model is less conservative as it has a smaller capacity as also shown in Figure 6b) which compares the various capacity curves between the four block studied, normalized with respect to block 4 .
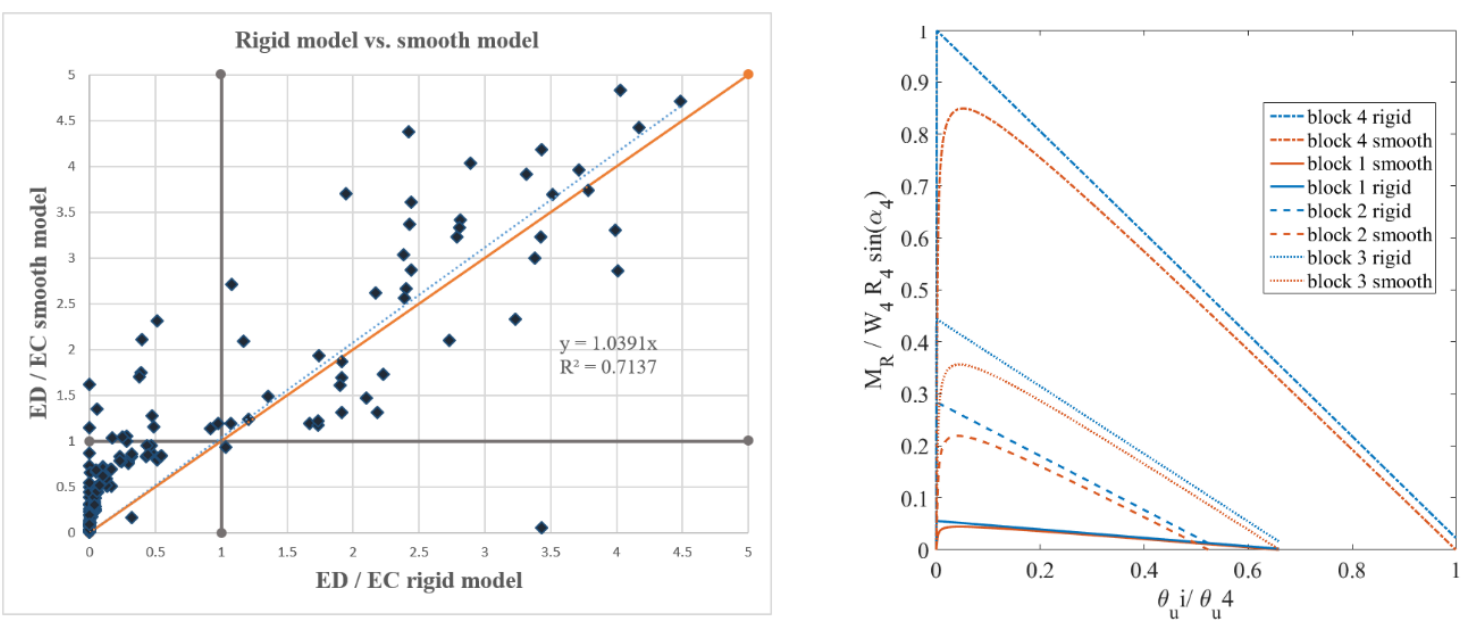

Figure 6. Comparison between the smooth and rigid model in terms of demand/capacity ratios (a) capacity curves for the four blocks considered (b). 
In the following, the behavior of masonry walls subjected to out-of-plane loads predicted by the proposed model is compared to the one predicted by the force-based (fig. 7a) and displacement-based procedures (fig. 7b) reported in the Italian code [10]. The discussion of the results is made in terms of conservativeness of the procedure and scatter of the response, measured through the coefficient of correlation $\mathrm{R}^{2}$ for a linear regression passing through the origin. When neglecting the results in which the block overturns for both numerical analysis and code-based procedures, force and displacement-based approaches seem very conservative while the energy-based approach (see fig. 8) seems less conservative also showing a slightly less scatter response. Currently there is a considerable scatter between non linear numerical analyses and simplified approaches models offset by adequate safety margins. This confirms the fact that the non-linear analysis prove valuable for a more physical representation of the phenomenon and also that the accuracy of the predictions using linear elastic spectra for rocking structures will always be very limited [13].

Anyway, further numerical analysis, considering a wider range of signals and blocks, should be done to better assess the level of reliability of the approaches proposed in the Italian code [10].
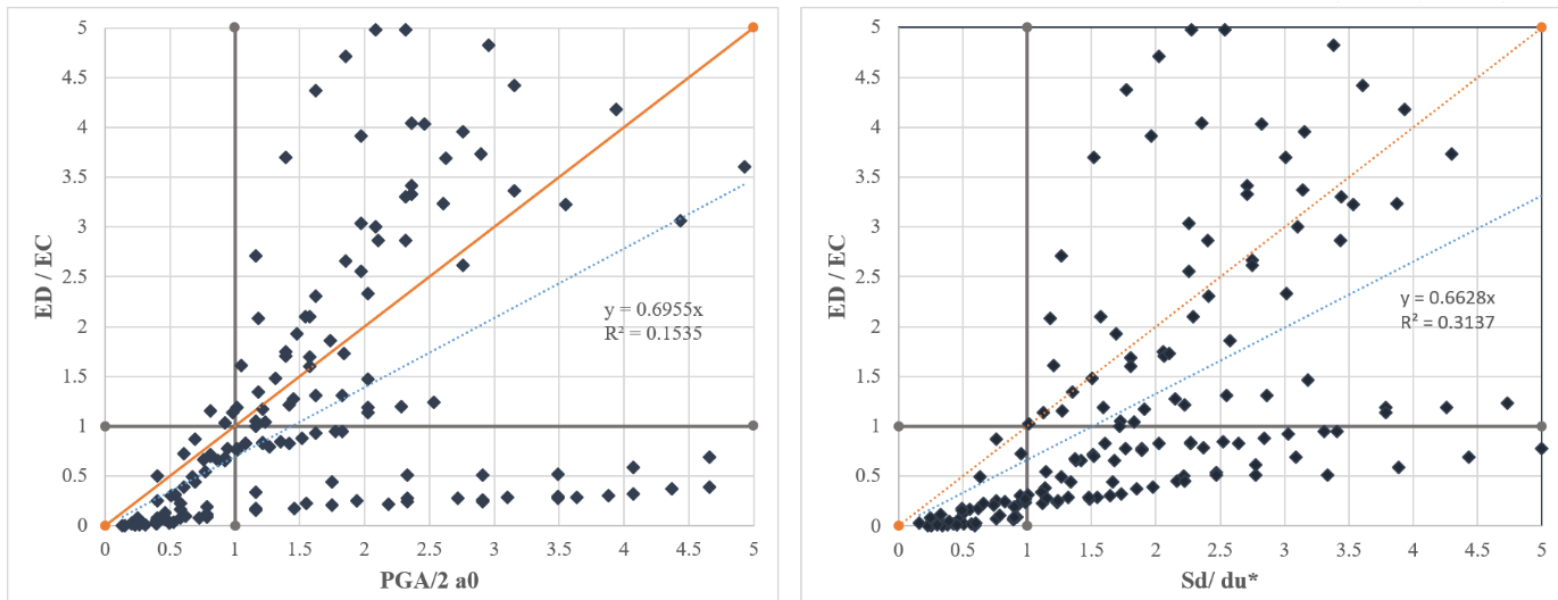

Figure 7. Comparison between the smooth model and code demand/capacity ratios (a) force-based and (b) displacement-based.

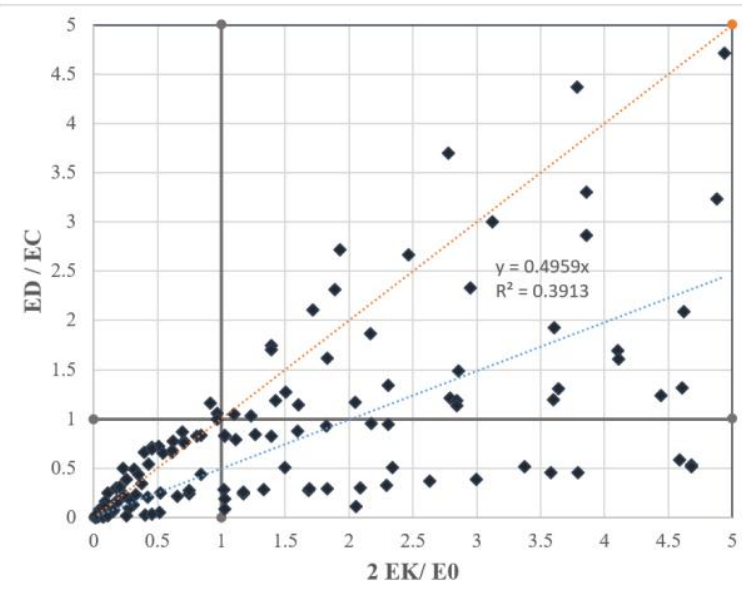

Figure 8. Comparison between the smooth model and the energy-based approach. 


\section{CONCLUSIONS}

- In this paper, a smooth moment-rotation relationship for describing the response of a block subjected to rocking motion is presented and the most significant aspects of its behavior have been highlighted.

- The replicated experimental tests have evidenced that the model is able to capture quite well the skeleton curve of the block in static conditions and the response under free oscillations, taking into account the stiffness of the foundation.

- Preliminary results suggest that the model may be able to be adopted for simulating the behavior of walls with loss of monoliticity, but this issue should be still addressed in detail.

- Comparing the results of the parametric analysis in terms of energy based ratios between the rigid and smooth block, it seems that the latter could be a good model to describe the behavior of rocking structures given that it reacts also in the field of small oscillations. Further numerical analysis should be done to better assess the reliability of code approaches.

\section{REFERENCES}

[1] I.N. Psycharis, Dynamic behaviour of rocking structures allowed to uplift, California Institute of Technology, Pasadena, California, 1982.

[2] GW Housner, The behaviour of inverted pendulum structures during earthquakes. Bull Seismol Soc AM 1963;53(2):402-17.

[3] A. Mauro, G. de Felice, M. J. DeJong, The relative dynamic resilience of masonry collapse mechanisms. Engineering Structures, 85, 182-194, 2015. DOI:10.1016/j.engstruct.2014.11.021.

[4] K. Doherty, M. Griffith, N. Lam, J. Wilson, Displacement-based analysis for out-ofplane bending of seismically unreinforced masonry walls. Earthquake Engineering and Structural Dynamics 2001; 31 (4): 833-50.

[5] O.A. Shawa, G. de Felice, A. Mauro, L. Sorrentino, Out-of-plane seismic behaviour of rocking masonry walls. Earthquake Engineering and Structural Dynamics, 41, 949-968, 2012. DOI: $10.1002 /$ eqe. 1168 .

[6] T.M. Ferreira, A. A. Costa, R. Vicente, H. Varum, A simplified four-branch model for the analytical study of the out-of-plane performance of regular stone URM walls. Engineering Structures, 83 (2015) 140-153.

[7] E. Dimitrakopoulos, M.J. DeJong, Revisiting the rocking block: closed-form solutions and similarity laws. Proc R Soc A 2012:468:2294-318.

[8] G. de Felice, Out-of-plane seismic capacity of masonry depending on wall section morphology. Int J Architect Herit 2011; 5 (4-5):466-82. 
[9] M.A. ElGawady, Ma Quincy, J.W. Butterworth, J. Ingham, Effects of interface material on the performance of free rocking block. Earthquake Engineering and Structural Dynamics 2011; 40(4):375-392.

[10] Consiglio Superiore dei Lavori Pubblici. Circolare 02 febbraio 2009 n.617 Istruzioni per l'applicazione delle "Nuove norme tecniche per le costruzioni" di cui al DM 14 gennaio 2008. Gazzetta Ufficiale della Repubblica Italiana n.47, Supplemento Ordinario n.27, C8.A.4. Roma 2009; (in Italian).

[11] L. Sorrentino, D. DAyala, G. de Felice, M. Griffith, S. Lagomarsino, G. Magenes, Review of out-of-plane seismic assessment techniques applied to existing masonry building. International Journal of Architectural Heritage, submitted 2015.

[12] A. Costa, A. Arede, AA. Costa, TM Ferreira, A Gomes, H Varum. Experimental study of the out-of-plane behaviour of unreinforced sacco stone mansonry walls: Comparative analysis of two different test setups. In: $9^{\text {th }}$ International masonry conference - 9IMC, Paper ID: 1467, 7-9 July, Guimaraes, Portugal;2014.

[13] N. Makris, D. Konstantinidis. The rocking spectrum and the limitations of practical design methodologies. Earthquake Engineering and Structural Dynamics 2003; 32(2):265-89. 